\title{
Evaluación del proceso de transformación de la matriz productiva ecuatoriana
}

\section{Evaluation of the transformation process of the Ecuadorian productive matrix}

\author{
Ing. Elizabeth Arias Domínguez, Mgt. ${ }^{1}$ \\ eariasd@ulvr.edu.ec \\ Ec. Sergio Cristóbal Suárez López, Mgs. ${ }^{1}$ \\ ssuarezl@ulvr.edu.ec \\ Iris Tahita Minchala García ${ }^{1}$ \\ iminchalag@ulvr.edu.ec \\ Katherine Briggitthe Medina Livisaca ${ }^{1}$ \\ kmedinal@ulvr.edu.ec
}

Recibido: 1/12/2016, Aceptado: 1/2/ 2017

\begin{abstract}
RESUMEN
La economía de Ecuador se ha caracterizado por la exportación e importación de productos agrícolas, sin embargo, en los últimos años el gobierno ha tratado de cambiar este modelo, fomentando la transformación de la matriz productiva. Este tema se refiere a realizar varios cambios que implican la modificación global de los sectores y actividades con mayores demandas de un país; recordando que Ecuador se mantiene con una economía principalmente petrolera y agrícola; este proceso tiene como fin de innovar con productos industrializados dándole así valor agregado para que puedan competir en el exterior en las mismas condiciones. El modelo actual para el cambio de la matriz productiva tiene el enfoque de convertir a Ecuador en un país industrializado, con énfasis en la innovación y en la generación de conocimiento para no sólo enfocarse en productos primarios. El gobierno inició sus actividades fomentando estrategias como el Plan Nacional del Buen Vivir. Las acciones emprendidas actualmente, tratan de mejorar y crear un cambio a la visión del Ecuador, pero los precios internacionales han desequilibrado la balanza comercial del país. La transformación de la matriz productiva es a largo plazo y los esfuerzos deben ser constantes para poder observar dichos cambios.
\end{abstract}

Palabras clave: Exportación, importación, balanza comercial, matriz productiva, valor agregado

\footnotetext{
${ }^{1}$ Docente de la Universidad Laica Vicente Rocafuerte de Guayaquil. Ecuador
} 


\begin{abstract}
The economy of Ecuador has been characterized by the export and import of agricultural products, however in recent years the government has tried to change this model, encouraging the transformation of the productive matrix. This theme refers to making several changes that involve the global modification of the sectors and activities with greater demands of a country; Recalling that Ecuador is maintaining a mainly oil and agricultural economy; This process aims to innovate with industrialized products giving added value so that they can compete abroad under the same conditions. The current model for the change of the productive matrix has the focus of turning Ecuador into an industrialized country, with an emphasis on innovation and the generation of knowledge to not only focus on primary products. The government began its activities by promoting strategies such as the National Plan for Good Living. The actions currently undertaken seek to improve and create a change to the vision of Ecuador, but international prices have unbalanced the country's trade balance. The transformation of the productive matrix is long-term and efforts must be constant to be able to observe these changes.
\end{abstract}

Keywords: Export, import, trade balance, productive matrix, value added

\title{
Introducción
}

En el mercado internacional, Ecuador se ha caracterizado por muchos años en ser un país proveedor de materia prima y también por ser comprador de productos terminados con un alto valor agregado. El realizar cambios en la matriz productiva es darle un giro importante a la economía del país.

Este proceso sólo se lograría si se asignara la atención debida a cada sector productivo, capacitando a sus actores adecuadamente para que puedan realizar las transformaciones necesarias tanto en las industrias como en los bienes terminados y de esa forma se pueda incentivar la inversión extranjera, creando nuevas fuentes de empleo.

Desde hace algunos años, se ha tomado la iniciativa de ser competitivos por medio de la innovación y el desarrollo de nuevas estrategias basadas en la construcción del conocimiento.

Para realizar los cambios adecuados se debe tomar en cuenta ciertas interrogantes, por ejemplo: ¿qué, de qué forma y para quién producir?, con la finalidad de poder determinar hacia dónde dirigir el desarrollo de cada sector y cuál debería ser el aporte de cada uno de ellos para poder alcanzar una palpable transformación económica.

Para esto se debe determinar las necesidades que tienen los diferentes productores, dándoles la información necesaria, capacitarlos, poner a su alcance recursos económicos por medio de créditos accesibles, para que puedan llegar a nuevos mercados.

Transformar la matriz productiva es la meta a alcanzar más importante del país, pero no es un objetivo a corto plazo. Ecuador puede llegar a ser una nación más competitiva cristalizando cambios como la sustitución de importaciones y el 
crecimiento de las exportaciones mediante el desarrollo de la producción nacional, mejorando así los resultados de la balanza comercial.

En la última década se ha evidenciado la necesidad de cambiar el enfoque basado en los recursos naturales que produce Ecuador, sobre todo al volver a enfrentar crisis fruto de la dependencia a productos como el petróleo. Para poder alcanzar la sociedad del Buen Vivir se debe procurar producir artículos de vanguardia, alta tecnología y también servicios de buena calidad; existe el talento humano para cumplir esta meta, pero se lo debe complementar con una inversión permanente en educación e investigación.

Se requiere llegar a disminuir las importaciones de productos que el país pueda producir bajo su propia marca, "Primero Ecuador". El uso de ésta representa una gran responsabilidad porque para obtener la confianza del consumidor hay que ofrecer igual o mejor calidad del producto o servicio de las marcas internacionales y conocer las preferencias de los consumidores.

Lo que se quiere lograr es estimular las exportaciones de los productos ecuatorianos con valor agregado, para que estos sean reconocidos a nivel internacional y poder competir en iguales condiciones con los que ya se encuentran establecidos en el mercado mundial.

\section{Metodología}

Para realizar el presente estudio se efectuó una revisión bibliográfica y documental de fuentes oficiales y originales, por medio del análisis de la información de diferentes sitios web, para poder tener un panorama claro del tema a tratar.

En el documento realizado se aplicó el criterio objetivo y profesional de los autores, para emitir opiniones que realcen la importancia del tema tratado.

\section{Desarrollo}

\section{Matriz Productiva}

La matriz productiva es la forma cómo se organiza una comunidad o sociedad para producir determinados bienes, productos o servicios en un tiempo y a un precio determinado, esta no se limita únicamente a los procesos estrictamente técnicos o económicos, sino que también tiene la obligación de velar por esos procesos y realizar interacciones entre los distintos actores: sociales, políticos, económicos, culturales, entre otros, que utilizan los recursos que tienen a su disposición para llevar adelante las actividades de índole productivo (Diario Crónica, 2015).

La primera fase del cambio de la matriz productiva implica crear las condiciones económicas adecuadas. Ecuador ha enfocado sus esfuerzos en generar las situaciones óptimas para impulsar y mejorar la competitividad sistémica, con el objetivo de incrementar la productividad del país.

Estos avances son evidentes en temas de vialidad, financiamiento de la inversión productiva, clima de negocios, impulso a las compras públicas, promoción del comercio exterior sostenible, impulso a la agenda regulatoria, alianzas público privadas, acceso a tecnologías de información, mega construcciones, fortalecimiento 


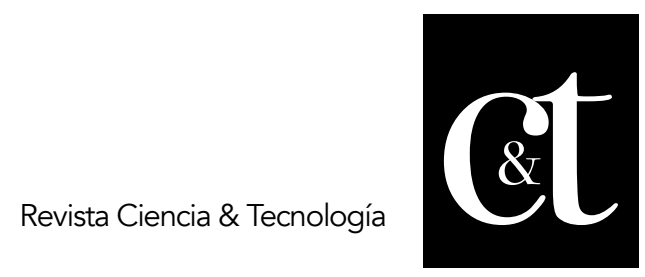

No.14, 30 de abril de 2017

ISSN impreso: 1390 - 6321

del talento humano y fomento a la cultura de emprendimientos.

Estas bases servirán como eje para el repunte de la agroindustria, las industrias básicas, intermedias y finales, y los servicios, consolidando un sistema productivo basado en la eficiencia e innovación (Senplades, 2017).

Ecuador busca la industrialización de los sectores primarios para avanzar a un producto que contenga valor agregado para generar desarrollo y progreso, esta definición incluye muchos aspectos tanto cuantitativos incidiendo en los procesos económicos de cada sector productivo como cualitativos viendo los aspectos políticos que van relacionados hacia una misma meta.

La matriz productiva se refiere a ampliar la cantidad de industrias tanto de insumos como de bienes finales con el fin de agrandar el aparato productivo ecuatoriano. (Diario Crónica, 2015), por lo que el gobierno está incentivando a los productores a hacer más allá de una simple cosecha, sino que ahora ofrezcan a sus compradores un producto ya terminado que no solo beneficiará al país generando empleo adecuado, sino también a ellos, incrementado sus ingresos.

Plan nacional del Buen Vivir

El Plan Nacional para el Buen Vivir 2013-2017 es un plan a escala nacional. Está compuesto de un conjunto de 12 objetivos, que se detallan en la tabla 1.

Estos objetivos expresan la voluntad de continuar con la transformación histórica del Ecuador. Este documento y su aplicación se crearon bajo la consigna de ser un referente en Latinoamérica.

Sin embargo, los objetivos que sustentan el importante tema abordado en este estudio son los dirigidos a mejorar la calidad de vida de los ecuatorianos, al surgimiento y crecimiento del sistema económico solidario y de la transformación positiva de la matriz productiva, específicamente los objetivos 3 (mejorar la calidad de vida de los hombres y mujeres ecuatorianos), 8 (consolidar el sistema económico social y solidario, de forma sostenible) y 10 (fomentar la transformación de la matriz productiva). 


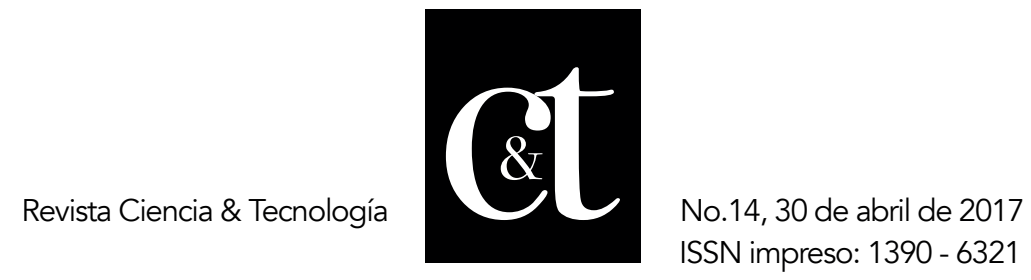

\section{Tabla 1. Los objetivos del Plan Nacional del Buen Vivir}

Objetivo 1: Consolidar el Estado democrático y la construcción del poder popular.

Objetivo 2: Incentivar la igualdad, la cohesión, la inclusión y la equidad social y territorial, en la diversidad.

Objetivo 3: Mejorar la calidad de vida de los hombres y mujeres ecuatorianos.

Objetivo 4: Fortalecer las capacidades y potencialidades de los ciudadanos.

Objetivo 5: Crear espacios de encuentro común y fortalecer la identidad nacional, las identidades diversas, la plurinacionalidad y la interculturalidad.

Objetivo 6: Transformar la justicia y fortalecer la seguridad integral, en estricto respeto a los derechos humanos.

Objetivo 7: Garantizar los derechos de la naturaleza y promover la sostenibilidad ambiental territorial y global.

Objetivo 8: Consolidar el sistema económico social y solidario, de forma sostenible.

Objetivo 9: Garantizar el trabajo digno.

Objetivo 10: Fomentar la transformación de la matriz productiva.

Objetivo 11: Garantizar la soberania y eficiencia de los sectores estratégicos para la transformación industrial y tecnológica.

Objetivo 12: Asegurar la soberanía y la paz, impulsar la inserción estratégica en el mundo y la integración latinoamericana.

Fuente: Secretaría Nacional de Planificación y Desarrollo (2013) 


\section{Sectores prioritarios}

Se han identificado 14 sectores productivos y 5 industrias estratégicas para el proceso de cambio de la matriz productiva de Ecuador, los mismos que se pueden apreciar en la tabla 2 (Secretaría Nacional de Planificación y Desarrollo, 2013). El fortalecimiento de estos sectores ayudará a lograr un crecimiento económico sostenido; ya que la creación de emprendimientos y la inversión en este tipo de industrias incrementará el nivel de empleo, que es una de las necesidades más urgentes que presenta la sociedad ecuatoriana.

Tabla 2. Sectores Prioritarios

Sector

\section{Industria}
Bienes
1) Alimentos frescos y procesados
2) Biotecnologia (bioquimica y biomedicina)
3) Confecciones y calzado
4) Energias renovables
5) Industria farmacéutica
6) Metalmecánica
7) Petroquímica
8) Productos forestales de madera
Servicio 9) Servicios ambientales
10) Tecnologia (software, hardware y servicios informáticos)
11) Vehículos, automotores, carrocerias y partes
12) Construcción
13) Transporte y logistica
14) Turismo

Fuente: Secretaría Nacional de Planificación y Desarrollo, 2013

\section{Balanza Comercial}

La balanza comercial es un registro de las importaciones y las exportaciones de una nación, dentro de estas se pueden encontrar los productos tradicionales y no tradicionales.

En el gráfico 1 se encuentran las exportaciones de los productos no tradicionales en base a los datos generados por el Banco Central del Ecuador. 


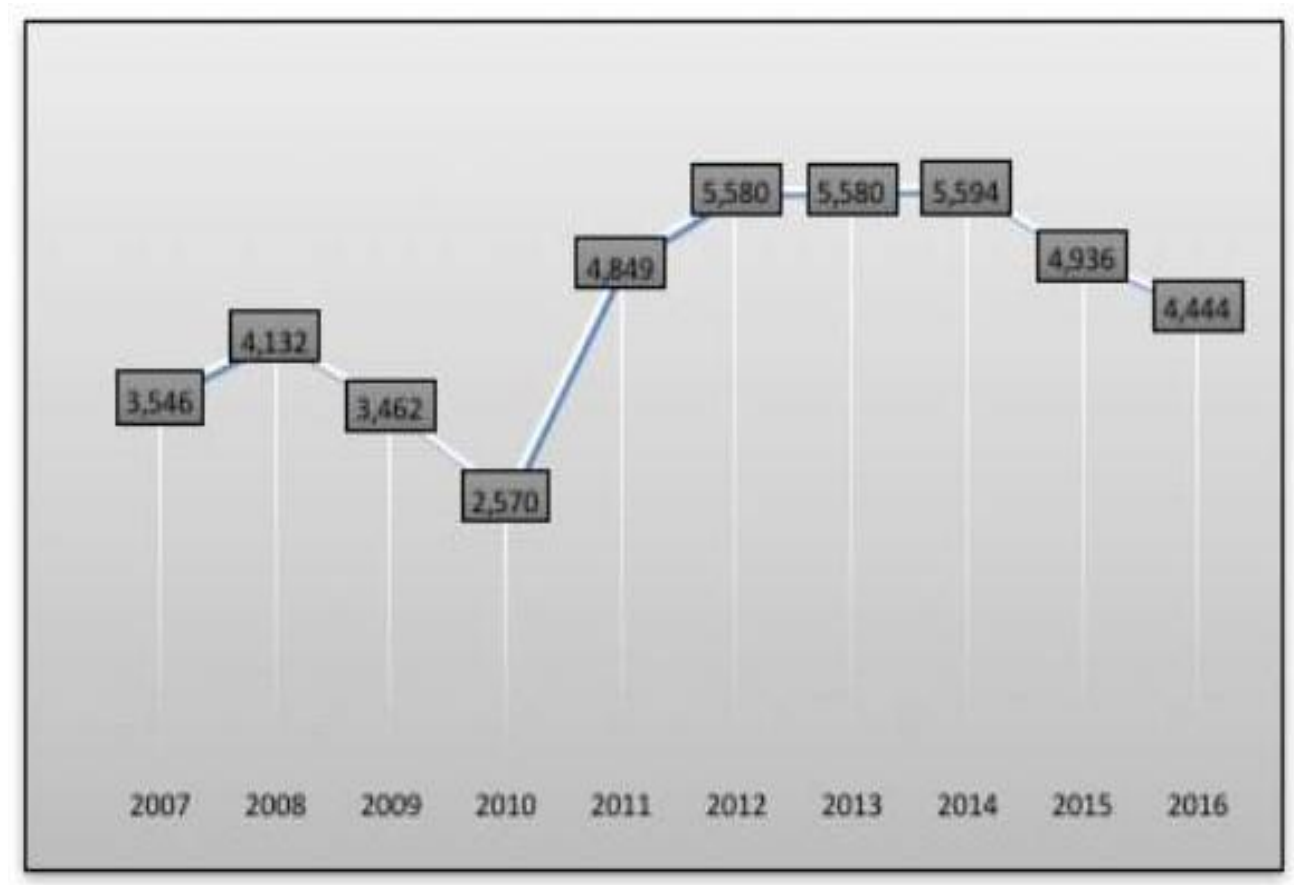

\section{Gráfico 1. Exportación total de productos no tradicionales por año (Millones USD FOB)}

Fuente: Banco Central del Ecuador (2016)

Ecuador siempre se ha especializado en exportar materia prima, con un número reducido de productos y a una cantidad mínima de destinos. Debido a esto, surge la necesidad de impulsar la exportación de productos no tradicionales. Se puede observar en el gráfico 1, la balanza comercial de los productos no tradicionales comienza con 3.546 millones de dólares en el año 2007 (Banco Central del Ecuador, 2016), periodo inicial la presidencia del Econ. Rafael Correa, es el punto de partida del cambio de la matriz productiva. En lapso de un año se vieron respuestas positivas al Plan Nacional del Buen Vivir, por lo que se vio un incremento del $16 \%$ en el 2008. Pero para los años 2009 y 2010 se presentó un notorio déficit. Desde el año 2011 al 2014 se evidenció un comportamiento creciente, hasta que en el 2015 y 2016 se registraron decrementos, fiel reflejo de la recesión económica que se sufrió. 


\title{
Tabla 3. Análisis de los productos industrializados
}

\begin{abstract}
Alimentos
85.900 empleos se generaron desde este sector, aportando en la balanza procesados: comercial de manera positiva, cumpliendo con el objetivo de sacar al mercado bienes terminados diferenciados por la calidad de la materia prima con la que se producen.

\section{Confecciones y}

Se ha notado un claro incremento pero no el óptimo, por la competencia Calzados: desleal del mercado, pero la tarea de este sector es mejorar aún más la calidad para seguir creciendo, teniendo en cuenta que el consumo de calzado del ecuatoriano es de 2,5 pares promedio.
\end{abstract}

Biotecnología Se necesita brindar el apoyo necesario a las carreras médicas en las principales (Bioquimicay universidades de Ecuador, para que puedan desarrollar nuevas Biomedicina): investigaciones.

Energias Renovables:

\section{Industria} Farmacéuticas:

Metalmecánica:

Petroquimica:

Productos forestales de madera:

Construcción:
Se contó con la inversión de \$ 43,4 millones en el proyecto eólico villonaco, este es un sector demasiado importante ya que de este se derivan varios procesos productivos.

$\$ 300$ millones en importaciones se han reducido gracias a las mejoras que se les ha dado a este sector logrando ocupar casi el $25 \%$ del mercado actualmente.

Es un sector importante para el cambio y también genera empleo, claro ejemplo de esto es el cambio a las cocinas de inducción aunque no está del todo aceptado por los ciudadanos, pero se han producido 230.000 unidades de estas lineas blancas.

Se generaron más de 20.000 empleos gracias a la construcción de la Refineria del Pacifico.

Aparte de generar empleo en este sector, también es priorizado, la innovación se da en los productos terminados, se sembraron 120.000 hectáreas de 15 especies forestales.

Este sector creció en $8,6 \%$ gracias a la importante inversión pública para las edificaciones que se fueron dando durante este periodo presidencial.

Fuente: Ekos Negocios, 2014. 


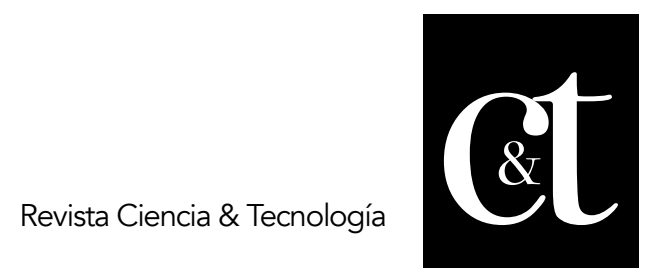

No.14, 30 de abril de 2017

ISSN impreso: 1390 - 6321

En la tabla 3, se muestra un análisis y aspectos más importantes de los productos industrializados que se han desarrollado con el cambio de la matriz productiva, durante el periodo presidencial del Econ. Rafael Correa.

Este cambio lleva un proceso a largo plazo, lo que se va dando es un giro no tan drástico en la economía y se desea asegurar una estabilidad sostenida, por lo que los resultados de esta transformación también se ven poco a poco y todos los esfuerzos realizados deben ser constantes, sin importar la tienda política de los mandatarios; para poder aprovechar la inmensa riqueza de lo tradicional en Ecuador, el enorme potencial que tiene en su campo y a todo esto añadirle el valor adicional que da la tecnificación para que su comercialización traiga mayores beneficios al territorio ecuatoriano.

Durante el periodo del presidente Rafael Correa se motivó a consumir primero lo ecuatoriano, reduciendo las importaciones que se hacían de productos extranjeros, claro está que procurando contar con una mano de obra más calificada y una mejor tecnología para dejar de priorizar ser un país proveedor de materia prima.

El gobierno de la revolución ciudadana ha logrado objetivos muy importantes para el desarrollo de Ecuador, pero para llevar a cabo este gran proyecto es necesario recorrer un largo camino, se debe contar con la ayuda y la coordinación de algunas instituciones como las que se mencionan a continuación:

- MCPEC Ministerio de Coordinación de la Producción, Empleo y Competitividad

- MCPE Ministerio Coordinador de Política Económica. (Secretaría Nacional de Planificación y Desarrollo, 2013)

Con la ayuda de estas instituciones se gana competencia en los mercados, pero así también se han ido sumando más exigencias de calidad en los productos, por lo cual debe existir siempre una coordinación entre todos los protagonistas en las diferentes actividades comerciales.

Son innegables los esfuerzos emprendidos por transformar la forma de producir y pensar en Ecuador, pero es el comienzo de un camino largo por recorrer. Además, de disponer de la infraestructura que se requiere para generar costos que dinamicen el mercado y el buen desempeño de las actividades productivas, también se debe poner atención en los cuatro ejes fundamentales de la matriz productiva.

Primer eje: diversificación de la producción, es decir, ofrecer nuevos productos a los mercados, productos ya procesados por ende con valor agregado ampliando el mercado y a su vez con nuevos productores/empresas.

Segundo eje: Valor agregado, lo que se ha explicado a lo largo de este artículo, incentivar a los productores y agricultores de vender más allá que solo la materia prima sino también vender un producto procesado con componentes de tecnología e innovación. 
Tercer eje: Sustitución selectiva de importaciones, aquí nace el incentivar a consumir primero lo nuestro y no lo extranjero, reduciendo el porcentaje de importaciones de Ecuador, para ello los productores deberían contar con la tecnología necesaria para empezar a producir muchas de las cosas que se traen de otros territorios.

Cuarto eje: Mejorar la oferta exportable y lograr una mayor productividad, este eje se va a complementar con el cumplimiento de mayores estándares de producción, tecnología, eficiencia y rapidez.

Al basarse estrictamente en los cuatro ejes fundamentales de la matriz productiva, ya explicados con anterioridad, claramente se puede notar que han existido esfuerzos para lograr esta transformación, pero el objetivo principal tardaría en que se lo cumpla.

Comenzando por el primer eje, que va encadenado con el cuarto, no se han desarrollado en gran parte porque para poder ofrecer mejor calidad en los productos o diversificación de los mismos, se necesita tecnología avanzada y para la constitución de nuevas empresas se requiere también facilidad para acceder a créditos.

Al segundo y tercer eje son a los que más pesos se les ha dado, porque a diario se puede palpar la motivación a los ciudadanos para consumir lo nacional. De esta forma se orientan a los pequeños y grandes productores en el cambio de la matriz productiva, para que no solo las materias primas se exporten sino también la industrialización de los productos, dándole así valor agregado con la finalidad de disminuir el consumo de bienes extranjeros, que también es cierto, muchos de ellos están hechos con materia prima ecuatoriana.

\section{Conclusiones}

La transformación de Matriz Productiva y el Plan Nacional del Buen Vivir, comprenden objetivos, planes y metas creados con la finalidad de trasladar los productos del mercado ecuatoriano fuera de sus fronteras. Al transcurrir del tiempo, desde el periodo que se inició con este proyecto se han visto cambios, si bien se sabe que este proceso no se da a corto plazo, en la actualidad todavía quedan asuntos pendientes.

También hay que darle énfasis en lo que tecnología se trata, ya que eso es uno de los puntos clave en el segundo y tercer eje de la matriz productiva. Los productores se han ido especializando en lo que a bienes procesados se trata, pero todavía hay mucho que aprender, crear e innovar.

La tecnología con la que cuenta Ecuador todavía es muy básica en comparación con otros países de Latinoamérica y del mundo, se necesita el apoyo del Estado para financiar maquinaria de alta generación y transferencia de conocimientos, además de la inversión de empresas extranjeras para entre todos se colabore con este proceso de transformación.

Ecuador es un país con una gran riqueza de productos agrícolas; sin embargo, se necesita un gran aporte en tecnología para competir en similares condiciones en los mercados extranjeros. La tendencia a nivel internacional es darle mayor valor a los 


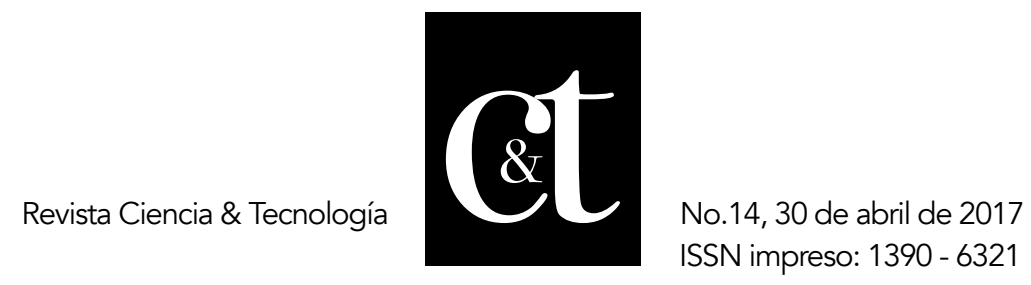

productos de alta generación y vanguardia, que se crean mediante un conjunto de varios componentes como: la innovación, el emprendimiento y las estrategias empresariales que pueden llevar al éxito a una empresa y a su vez a un país.

En el proceso de la trasformación de la matriz productiva se debe dar especial atención a la motivación que requieren los agricultores y productores de materia prima, para que den el siguiente paso que es especializarse en productos terminados de mejor calidad.

Está claro que Ecuador necesita una economía de escala, es decir que debe vender en grandes cantidades a grandes mercados; para esto se tiene ventajas como el acuerdo recientemente firmado entre la Unión Europea, recalcando que esta región es unos de los más grandes mercados a nivel mundial al igual que Estados Unidos; aunque siempre es válido ir en la búsqueda de nuevos destinos comerciales.

\section{Referencias bibliográficas}

Banco Central del Ecuador (2016). Banco Central del Ecuador. Recuperado el 12 de 05 de 2017, de https://www.bce.fin.ec/index.php/component/k2/ item/756

Diario Crónica (26 de 08 de 2015). Crónica. Recuperado el 05 de 02 de 2017, de http://www.cronica.com.ec/ opinion/columna/columnista/item/7646-lamatrizproductiva

Ekos Negocios. (noviembre de 2014). Ekosnegocios. Recuperado el 01 de 02 de 2017, de http://www.ekosnegocios.com/revista/ pdfTemas/1091.pdf

Secretaría Nacional de Planificación y Desarrollo (2013). Plan Nacional del Buen Vivir. Recuperado el 12 de 05 de 2017, de http://www.buenvivir.gob.ec/

Secretaría Nacional de Planificación y Desarrollo Senplades (2017). Recuperado el 12 de 05 de 2017, de http://www.planificacion.gob.ec/wpcontent/ uploads/downloads/2017/04/Informe-a-la-Nacion.pdf

Secretaría Nacional de Planificación y Desarrollo (2013). Transformación de la Matriz Productiva.Recuperado el 02 de 02 de 2017, de http://www.planificacion.gob.ec/wp- content/uploads/ downloads/2013/01/matriz_productiva_WEBtodo 